\title{
Photo-assisted VOC Detection by Transparent pn Junction
}

\author{
Yoshinobu Nakamura $^{1}$, Yusuke Morita ${ }^{2}$, Yasuhiro Yoshida ${ }^{2}$, and Satoru Fujitsu ${ }^{2}$ \\ ${ }^{1}$ Department of Advanced Materials Science, School of Frontier Science, The University of Tokyo \\ 5-1-5 Kashiwanoha, Kashiwa, Chiba, 277-8561, Japan \\ ${ }^{2}$ Department of Materials Science and Ceramic Engineering, Shonan Institute of Technology \\ 1-1-25 Tsujido-nishikaigan Fujisawa, Kanagawa 251-8511, Japan
}

Keywords: Heterojunction, VOC, sensor, $\mathrm{NiO}, \mathrm{ZnO}$, photo-excitation, concert mechanism

\begin{abstract}
The $p-\mathrm{NiO} / n-\mathrm{ZnO}$ transparent pn heterojunction is prepared by $\mathrm{RF}$ magnetron sputtering technique and processed into a chemical sensor element by photolithography pattering and chemical etching process. Forward biased $p-\mathrm{NiO} / n-\mathrm{ZnO}$ heterojunction sensor can detect VOCs at room temperature and its gas sensitivity is extremely enhanced with the assist of deep UV light incidence. Even if the testing gas is thermodynamically stable such as aromatic VOC, the $p-\mathrm{NiO} / n-\mathrm{ZnO}$ heterojunction sensor can detect it with the concert mechanism involving "electric" and "photo" assistance.
\end{abstract}

\section{INTRODUCTION}

Detection and monitoring technique for VOC (Volatile Organic Compound) had been desired from the viewpoint of maintenance of safe environment. The simplest solid-state device for VOC detection is a semiconductor gas sensor and its working mechanism is the change in surface resistance followed by the catalytic oxidation of combustible gases in atmosphere. However, thermodynamically stable aromatic VOCs hardly interact with the surface of the solid-state devices and they cannot be detected by a simple gas sensor element. How to activate the thermodynamically stable molecules is a key for designing a solid-state VOC sensor. For this purpose, the authors will propose a new concept of a pn junction type VOC sensor, whose working mechanism is based on the assistance of "external" energy, that is, electric potential energy or photo-energy.

When VOC molecules are introduced into the pn junction interface having "open" structure shown in Fig.1, the junction region works as a catalyst and their oxidation reaction will proceed. If forward d.c. bias is applied to the junction, holes in the p-type layer and electrons in the n-type layer are concentrated into the pn junction region, and they would interact with adsorbed VOC molecules. In our hypothesis, valence electrons of an adsorbed VOC molecule would be extracted due to the higher oxidation power of holes in p-type semiconductor, then the adsorbed molecule becomes unstable. Such an unstable molecules is easy to react with adsorbed oxygen near the adsorption sites, following the Langmuir-Hinselwood mechanism. When the adsorbed oxygen over the n-type layer is consumed by the oxidation reaction with VOC, the junction resistance decreases, while it increases when the adsorb oxygen over the p-type layer is consumed. The so-called vapor phase electro-catalytic reaction would proceed at the pn junction and acceleration of the catalytic reaction would be expected. In fact, the authors got an indirect evidence for the acceleration of $\mathrm{CO}$ oxidation reaction at the biased $p-\mathrm{CuO} / n-\mathrm{ZnO}$ heterojunction[1].

In the present study, the $p-\mathrm{NiO} / n-\mathrm{ZnO}$ heterojunction is prepared and processed to a gas sensor element. Nickel oxide is a wide gap $\left(\mathrm{E}_{\mathrm{g}}=3.7 \mathrm{eV}\right)$ p-type semiconductor whose valence band maximum is $5.3 \mathrm{eV}$ below the vacuum level[2]. Oxidation power of holes is thought to be proportional to the energy of valence band maximum(VBM) and it is not sufficient for the full oxidation of aromatic VOC. For the room temperature detection of the thermodynamically stable VOC, such as toluene or benzene, another assist process by external energy will be needed. Our 
next idea is the use of UV light incidence for the activation of testing gas during the sensor operation. Deep UV light can directly activate $\pi$ conjugated electrons in aromatic ring and pumped them up to the excited state, $\pi^{*}$. $\pi^{*}$ electrons would be unstable and easy to be extracted by the catalyst. The concerted mechanism involving photo and electro assist process would make possible to room temperature detection of aromatic VOC by a transparent pn junction sensor.

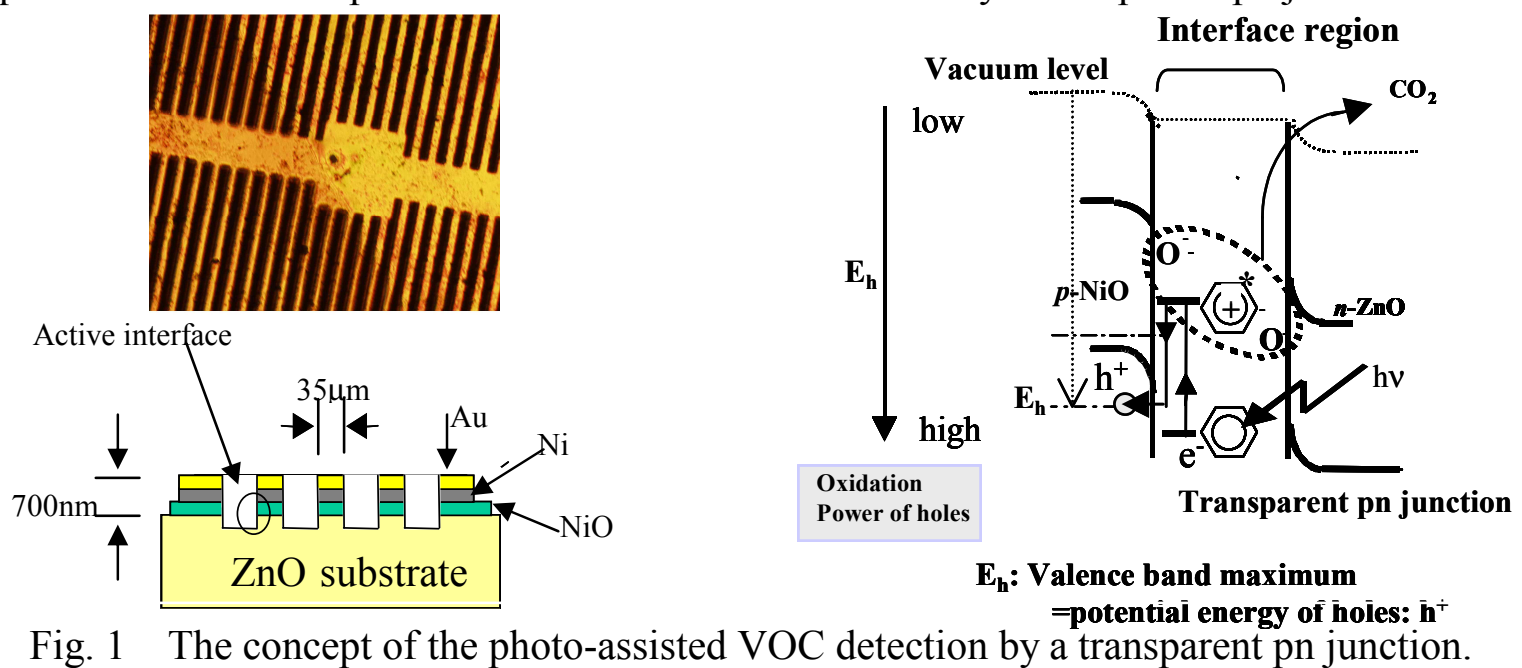

\section{EXPERIMENTAL PROCEDURE}

A highly oriented $\mathrm{ZnO}$ polycrystalline substrate was prepared by the vapor transport method. The grown $\mathrm{ZnO}$ polycrystalline was sliced parallel to the (0001) plane and the $\mathrm{ZnO}$ substrate having $\mathrm{Zn}$-terminated or O-terminated polar surface can be obtained. They were referred to as $\mathrm{Zn}$-face and $\mathrm{O}$-face, respectively and a substrate with $\mathrm{Zn}$-face was used for making a sensor element. NiO film of $500 \mathrm{~nm}$ thick was deposited on the $\mathrm{ZnO}$ substrate by the RF magnetron sputtering technique using the sputtering target of $2 \mathrm{~mol} \%$ lithium doped $\mathrm{NiO}$ sintered compact. The obtained $\mathrm{NiO}$ film was polycrystalline and no specific orientation of crystal axis was observed.

An Ohmic electrode, nickel metal film of $100 \mathrm{~nm}$ thick was soon deposited on the as-prepared $\mathrm{NiO}$ film and gold cover-electrode (100nm thick) was continuously deposited over the nickel metal electrode. For making the heterojunction specimen to atmosphere sensitive sensor element, the interface between $\mathrm{ZnO}$ substrate and $\mathrm{NiO}$ film must be exposed to the atmosphere. A comb-like photo-resist pattern, whose electrode was $35 \mu \mathrm{m}$ in width was drawn on the heterojunction specimen by photolithography technique and pre-etched it in $\mathrm{Ar}^{+}-\mathrm{O}_{2}^{+}$plasma to remove a part of upper electrode. Then the specimen was chemically etched in $0.5 \mathrm{M} \mathrm{HCl}$ aqueous solution and $\mathrm{NiO}$ layer not covered with resist pattern was completely removed. Wet etched specimen was plasma etched again and the $\mathrm{NiO}-\mathrm{ZnO}$ interface was completely exposed to the atmosphere as was shown in Fig.1.

The sensor element was set on the sample stage of an atmosphere controlled micro-probing system and tungsten probe was attached on the top electrode on $p$-NiO layer. The d.c. bias was applied to the junction and the current through the junction was monitored by d.c. current-voltage source/monitoring unit (Advantest TR-6143). The VOC was directly introduced into the atmosphere controllable sample stage by a $50 \mu \mathrm{l}$ micro-cylinde. Injected VOC was naturally vaporized and traveled to the junction interface with a carrier gas of $\mathrm{N}_{2}: \mathrm{O}_{2}=4: 1$. The VOC concentration around the specimen was predetermined by using a commercially available gas detector. UV-light source was a $17 \mathrm{~W}$ mercury vapor lamp, which was set over the sample stage.

\section{RESULT AND DISCUSSIONS}

Fig. 2 shows the current-voltage $(I-V)$ characteristics of the $p-\mathrm{NiO} / n-\mathrm{ZnO}$ heterojunction gas sensor element with and without acetone vapor introduction in atmosphere. The junction shows good rectifying character with the threshold voltage of $1.2 \mathrm{~V}$, which is a little larger than that predicted by the result of photoemission spectroscopy at the $p-\mathrm{NiO} / n-\mathrm{ZnO}$ interface: $0.54 \mathrm{~V}$ [2]. The 
$I-V$ curve shows hysteresis under the forward bias and that suggests the modified junction properties by the charged interface states. Current response by the introduction of acetone is also shown in Fig.2. The current changing process is rather complex. Immediately after the acetone injection, the current rapidly increases, then, gradually decreases in proportion to the increase in the acetone concentration. The mechanism of the rapid increase in current has not been understood yet, however, the possible mechanism is an additional ionic conduction through the liquid like acetone layer condensed at the junction interface. Only the forward current is modified by acetone vapor and it is suppressed according to the fluctuation of acetone concentration. This result suggests that the active zone of the $p-\mathrm{NiO} / n-\mathrm{ZnO}$ heterojunction sensor device is rectifying interface, not the bulk of $\mathrm{NiO}$ or $\mathrm{ZnO}$.

Room temperature gas sensing mechanism of the $p-\mathrm{NiO} / n-\mathrm{ZnO}$ heterojunction is guessed to be decrease in the amount of the surface/interface adsorbed oxygen with the assistance of high oxidation power of holes concentrated at the $p-\mathrm{NiO} / n-\mathrm{ZnO}$ interface. Gradual decrease in current in Fig. 2 supports our hypothesis that the adsorbed oxygen over $\mathrm{NiO}$ layer is consumed by the reaction with acetone. Similar gas sensing mechanism had already proposed on the $\mathrm{CO}$ gas sensing by the $p-\mathrm{CuO} / n-\mathrm{ZnO}$ heterojunction[1]. At room temperature, the oxidation reaction of organic compound over an oxide catalyst hardly proceed without the assist of external energy and in this case, "Electric" assist would be related to the working mechanism. Yanagida, et al. had reported the enhanced catalytic reaction of biased pn junction interface, and both the gas sensitivity and catalytic activity of the pn junction are enhanced in proportion to the applied bias[3]. However, in the room temperature operation, the contribution of electric filed assistance on the gas sensing characteristics is not so large and only small acetone sensitivity ( $8 \%$ current decrease by $4300 \mathrm{ppm}$ of acetone) is obtained at this $p-\mathrm{NiO} / n-\mathrm{ZnO}$ junction interface.

Fig. 3 shows the acetone sensing characteristics of the $p-\mathrm{NiO} / n-\mathrm{ZnO}$ heterojunction under deep UV light incidence. Similar to the result of Fig. 2, only the forward current decreases by the introduction of acetone but its sensitivity is four or five times higher than that worked in the dark. UV light incidence enhances the gas sensitivity of the $p-\mathrm{NiO} / n-\mathrm{ZnO}$ heterojunction and this result would be due to enhanced reactivity of photo-excited acetone molecules by deep UV light absorption. It is well known that absorption line of ketone is observed at $\lambda=330 \mathrm{~nm}$, which is assigned to $n-\pi^{*}$ transition. The UV source (a mercury vapor lamp) has line spectra of 254,313 , $340,365 \mathrm{~nm} \cdots$ and the line spectrum of $\lambda=313 \mathrm{~nm}$ and $340 \mathrm{~nm}$ is near the absorption lines of $\mathrm{n}-\pi^{*}$ (acetone) transition. If acetone in the excited state easily reacts with holes in $\mathrm{NiO}$, then react with adsorbed oxygen, the amount of surface adsorbed oxygen rapidly and largely decreases. Due to the concert mechanism involving the "electric-" and "photo-" assistance, the enhanced gas sensitivity of the $p-\mathrm{NiO} / n-\mathrm{ZnO}$ heterojunction are obtained.

The concert effect of "electric-" and "photo-" assistance will be more useful for the detection of more thermodynamically stable molecules, such as benzene or toluene. Without UV incidence, both forward and reverse current through the $p-\mathrm{NiO} / n-\mathrm{ZnO}$ junction interface did not change by the introduction of toluene or benzene vapor and electro-assist is judged to be invalid for toluene sensing in the dark. We suppose that the potential energy of holes in the valence band of $\mathrm{NiO}$ is insufficient for the oxidation of an aromatic ring of toluene. Binding energy of aromatic ring is evaluated to be $5.37 \mathrm{eV}$ and at most, more than $5.37 \mathrm{eV}$ of energy would be needed for the decomposition of toluene or benzene. However, under the UV light incidence, the forward current decreases according to the toluene introduction, and toluene sensing characteristics is restored.

Fig. 4 shows the toluene sensing characteristics of the $p-\mathrm{NiO} / n-\mathrm{ZnO}$ heterojunction under deep UV light incidence. The sensing characteristics are very similar to that of acetone sensing in Fig. 3 and similar working mechanism is suggested. Our hypothesis is as follows, toluene molecules are exited by $254 \mathrm{~nm}$ line of $\mathrm{Hg}$ lamp, because the absorption line of an aromatic ring is located at $\lambda=260 \mathrm{~nm}$, which is assigned to $\pi-\pi^{*}$ transition. Conjugated $\pi$ electrons are pumped up to $\pi^{*}$ orbital, then the molecules become unstable. Excited toluene molecules are oxidized or ionized by holes transported by the forward applied bias, then react with adsorbed oxygen over NiO layer. 
As a result, only the forward current responds to the fluctuation of toluene concentration. In the $p-\mathrm{NiO} / n-\mathrm{ZnO}$ heterojunction, the concert mechanism involving "electric" and "photo" assist plays an important role for the room temperature detection of aromatic VOC, toluene or benzene, which can never be detected by a traditional semiconductor gas sensor device.
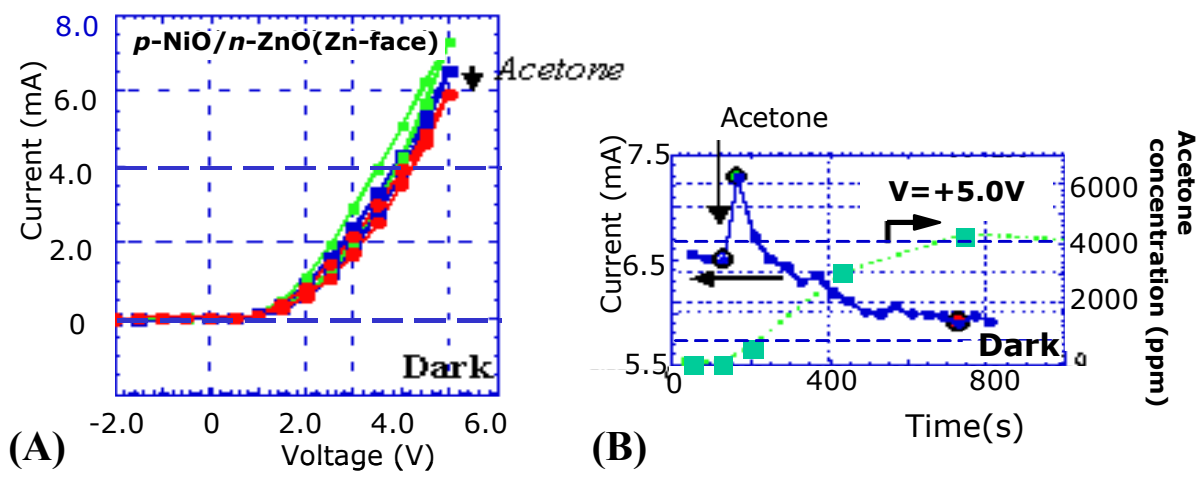

Fig.2 The effect of acetone introduction on (A) the I-V response and (B) the current-time behavior of the $p-\mathrm{NiO} / n-\mathrm{ZnO}$ heterojunction without $\mathrm{UV}$ incidence.

(A)

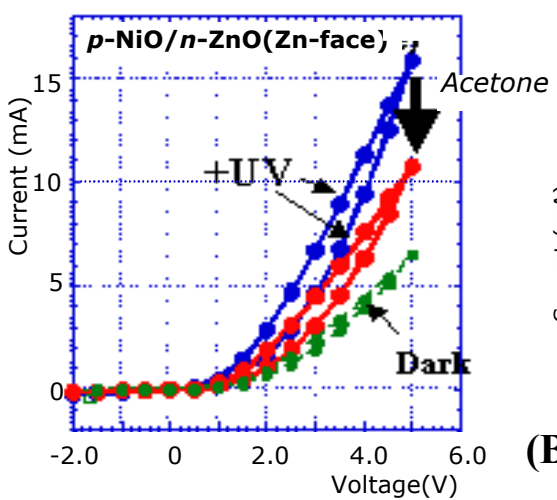

(B)

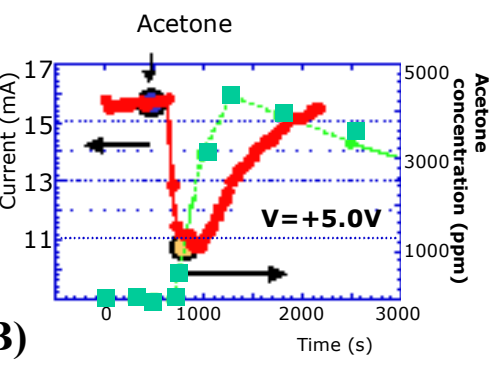

Fig.3 Enhanced acetone sensing characteristics of the $p-\mathrm{NiO} / n-\mathrm{ZnO}$ heterojunction by $\mathrm{UV}$ light incidence: (A) I-V response and (B) current-time behavior. UV source emits the line of $245 \mathrm{~nm}$.

(A)

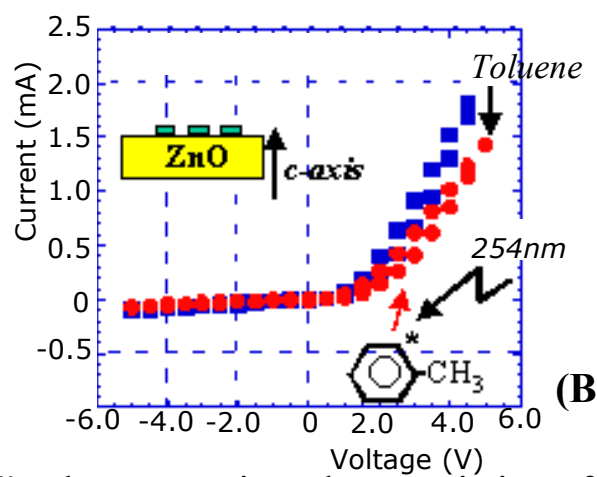

(B)

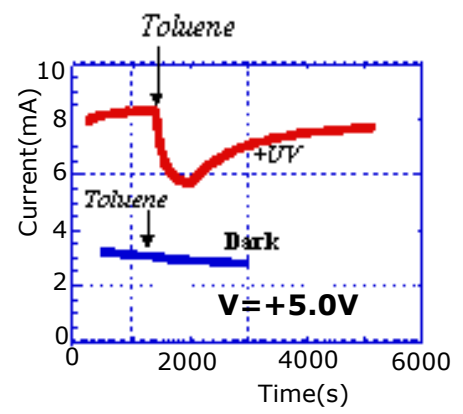

Fig.4 "Exotic" toluene sensing characteristics of the $p-\mathrm{NiO} / n-\mathrm{ZnO}$ heterojunction by UV light incidence: (A) I-V response and (B) current-time behavior. UV source emits the line of $245 \mathrm{~nm}$

\section{REFERENCES}

[1]Y.Nakamura, T.Tsurutani, O.Okada, Y.Nakamura, M.Miyayama, and H,Yanagida, J. Electrochem Soc., 137 (3) (1990), p.940

[2]Y.Ishida,A.Fujimori,H.Ohota,M.Hirano, and H.Hosono, Appl.Phys.Lett., 89 (2006),p.153502

[3] Y.Nakamura, Y.Ariga, A.Kishimoto, M.Miyayama, O. Okada, and H.Yanagida,

J.Electroceramics, 4:S1(1999),p105

e-mail: tnakamu@mail.ecc.u-tokyo.ac.jp

Fax: $\quad+81-4-7136-3792$ 